\title{
HABILIDAD EN PREGUNTAS CONCEPTUALES Y EN RESOLUCIÓN DE PROBLEMAS DE FÍSICA
}

\author{
LANG DA SILVEIRA, F.', MOREIRA, M.A. ${ }^{2}$ y AXT, R. ${ }^{2}$ \\ 1. Instituto de Física da UFRGS e Instituto de Física de la PUCRS. \\ 2. Instituto de Física de la UFRGS. Caixa Postal 1505l-Campus. 91500 Porto Alegre, RS, Brasil.
}

\begin{abstract}
SUMMARY
This study is described correlating the perfomance of engineering students in basically conceptual physics quizzes with their performance in physics exams constituted essentially by problem solving items. Research findings clearly suggest that mastery of concepts in a necessary but not sufficient condition for problem solving.
\end{abstract}

\section{INTRODUCCIÓN}

Para muchos alumnos, incluso en cursos universitarios introductorios saber física es, todavía hoy, una cuestion de conocer cuál es la «fórmula» que se debe aplicar para resolver un determinado problema. Naturalmente, esa visión distorsionada de lo que es saber física es una consecuencia directa de la enseñanza recibida, de la manera cómo se transmite el conocimiento (profesor, Iibro de texto, etc.) y de la forma cómo se evalúa al alumno (pruebas parciales, exámenes finales, ejercicios de final de capítulo, etc.). En todo este proceso se da, frecuentemente, una importancia exagerada a las fórmulas como punto de partida y no como el producto obtenido a partir de los conceptos físicos, su representación simbólica y su interdependencia.

Sin embargo, las cosas están cambiando. Este hecho es el resultado, principalmente, de los avances conseguidos por la investigación realizada en el área de los conceptos altemativos. Estos resultados sugieren, de forma inequívoca, que el conocimiento conceptual previo del alumno es factor determinante del aprendizaje futuro. Ese conocimiento muchas veces tiene significados distintos de los significados científicos (de ahí que se le llame alternativo). Por ello, en la actualidad, investigadores y profesores están realizando grandes esfuerzos intentando identificar estrategias, eficientes y viables, de cambio conceptual.

Es creciente el número de profesores preocupados ent comprender cómo los alumnos construyen conceptos, cómo los reformulan y perfeccionan, y cómo modelos y estrategias constructivistas pueden contribuir en ese sentido. Técnicas de enseñanza, esencialmente conceptuales, como la construcción de mapas conceptuales (Novak y Gowin 1984, Moreira y Buchweitz 1987), están siendo gradualmente introducidas día a día en las aulas. Además, están apareciendo libros de tex to con un enfoque acentuadamente conceptual, como es el caso, por ejemplo, de la obra «Conceptual Physics» (Hewitt 1987). La enseñanza en el laboratorio está siendo utilizada, también, como estrategia de cambio conceptual (Axt 1990).

Por otra parte, no puede haber exageración en el enfoque conceptual hasta el extremo de dejar de lado la solución de problemas en física o abandonar otros aspectos importantes de Ia enseñanza, considerando, por ejemplo, que una buena base conceptual es condición suficiente para convertir al alumno en un buen solucionador de problemas. Un toque de atención en ese sentido fue dado por Hellman (1898) en un trabajo en el que presenta un estudio comparando la capacidad de los alumnos en preguntas conceptuales y no conceptuales en exámenes de física. Hellman consideró no conceptuales las preguntas que implicasen únicamente sustiución de valores en formulas o que exigiesen únicamente la evocación de conocimientos memorizados. Como conclusión, proclamó la necesidad de intentar mantener un equilibrio entre preguntas conceptuales y no conceptuales en los exámenes de física, con el fin de evaluar de una forma real el aprendizaje del alumno. 


\section{EL ESTUDIO}

El estudio descrito en este trabajo es similar al de Hellman, en el sentido de comparar la capacidad de los estudiantes en preguntas conceptuales y no conceptuales. Hay, con todo, muchas diferencias:

1. Nuestro estudio es más amplio, incluyendo exámenes realizados por estudiantes de ingeniería de la UFRGS en asignaturas de física general, de 1985 a 1989 . La investigación de Hellman fue realizada con 130 estudiantes de física de una escuela secundaria norteamericana.

2. Las preguntas conceptuales que usamos pertenecían a exámenes que contenían un mínimo de 10 ítems, incluyendo algunos ya corregidos con anterioridad. Hellman usó únicamente dos preguntas conceptuales, de un total de 16 a 18 preguntas, en cada examen. Él reconoce este aspecto como una de las limitaciones de su estudio (op. cit., p. 385).

3. Por preguntas conceptuales entendemos, al igual que Hellman, aquéllas que implican la aplicación de conceptos, principios o leyes a determinadas situaciones físicas sin implicar substitución de valores en ecuaciones y cálculos numéricos o literales. Son preguntas cualitativas que implican comprensión y raciocinio frente a la simple memorización y habilidad de cálculo. Con todo, diferimos de Hellman en lo que respecta a preguntas no conceptuales. Como él mismo reconoce (ibid. p. 386), cuanto más avanzada está la carrera, más difícil es distinguir entre preguntas conceptuales y no conceptuales de la forma en que él lo hizo. Esto quiere decir que en cursos más avanzados raramente son propuestas preguntas de mera sustitución en fórmulas. En función de esto, en vez de preguntas no conceptuales optamos por usar el término preguntas de aplicación para designar aquéllas que comúnmente son llamadas preguntas de resolución de problemas.

4. Hellman sólo trabajó con porcentajes e histogramas, no teniendo en cuenta ni correlaciones, ni fiabilidad de los exámenes, ni la existencia de điferencias estadísticas significativas. Todos estos aspectos fueron considerados en nuestro estudio.

5. El objetivo de Hellman fue simplemente contrastar Ia hipótesis de que los estudiantes considerados mejores presentarian proporcionalmente mejores resultados en cualquier tipo de examen de física. La respuesta obtenida fue del tipo «depende» o «no necesariamente».

Nuestra hipótesis, enunciada a continuación, es diferente $\mathrm{y}$ tiene desdoblamientos.

\section{HIPÓTESIS}

Para resolver problemas (preguntas de aplicación), el alumno debe tener dominio de los conceptos relevantes. No es posible aplicar sin conocer los conceptos. En otras palabras, el dominio de los conceptos es una condición necesaria para su aplicación; sin embargo, no es una condición suficiente, ya que un alumno puede dominar la parte conceptual y no ser capaz de aplicarla.

Por esta razón, dados dos exámenes, presentados a los mismos alumnos, siendo uno de ellos conceptual (C) y el otro de resolución de problemas o de aplicación (A) se espera que:

1) Puntuaciones A elevadas no estén asociadas con puntuaciones $\mathrm{C}$ bajas.

2) Si se comparasen los grupos de individuos con $A$ elevado (grupo superior) y A bajo (grupo inferior), la media de las puntuaciones $C$ en el primero sería mayor que en el segundo. Esto implica la existencia de un coeficiente de correlación positivo entre $\mathrm{C}$ y $\mathrm{A}$.

3) Si fuesen comparados los grupos de individuos con $\mathrm{A}$ elevado (grupo superior) y A bajo (grupo inferior), la varianza de las puntuaciones $C$ en el primer grupo sería menor o igual que la del segundo grupo. Debe ser así, pues las puntuaciones A superiores no pueden estar asociadas a puntuaciones $C$ inferiores, pero puntuaciones $\mathrm{A}$ inferiores pueden estar asociadas a cualquier puntuación $\mathrm{C}$.

Estas hipótesis son válidas para puntuaciones $\mathrm{C}$ y $\mathrm{A}$ no falibles (sin error de medida o perfectamente fidedignas). Las puntuaciones $\mathrm{C}$ y A realmente obtenidas son siempre falibles $y$, por lo tanto, pueden existir violaciones de las hipótesis formuladas. Esas violaciones deberán ser pequeñas si los coeficientes de fidedignidad de las puntuaciones $\mathrm{C}$ y $\mathrm{A}$ fuesen razonables.

\section{METODOLOGÍA}

Con el objeto de validar las hipótesis enunciadas anteriormente se puede utilizar la siguiente metodología:

1 etapa: Calcular, en pares de exámenes $\mathrm{C}$ y $\mathrm{A}, \operatorname{los}$ coeficientes de fidedignidad respectivos (coeficiente alfa de Cronbach). Cuando los coeficientes son mayores de 0,60 se pasa a las etapas siguientes; cuando los coeficientes resultan inferiores a 0,60 no se sigue el estudio con esas puntuaciones, ya que por debajo de ese valor la fiabilidad del instrumento dejaría mucho que desear. (De ocho pares de exámenes inicialmente estudiados, uno fue desechado.)

$2^{\circ}$ etapa: Calcular el coeficiente de correlación entre C y A en cada par de exámenes, y su nivel de significación estadístico.

3etapa: Con base en la media y la desviación estándar de las puntuaciones $\mathrm{A}$ se clasifican como pertenecientes al grupo superior (A altos) o grupo inferior (A bajos). Los grupos inferiores y superiores están constituidos por las puntuaciones que se alejan de la media en más de $0,67 \mathrm{de}$ 
como consecuencia de Ia regresión estadística. En nuestro trabajo hemos tenido en cuenta las fiabilidades, ya que si éstas fuesen bajas el resultado sería trivial: regresión estadística.

\section{CONCLUSIÓN}

En la introducción de este trabajo destacamos, de modo muy positivo, una tendencia actual en la enseñanza de la Física que trata, por ejemplo, de enfatizar el aprendizaje de conceptos en detrimento de la memorización de fórmulas. A pesar de todo, llamamos también la atención sobre el hecho de que ese énfasis no debería llegar al extremo de descuidar otros aprendizajes que tradicionalmente se espera alcanzar en la enseñanza de la Física, como es el caso de la resolución de problemas. Además, este énfasis no debería llevar a la suposición de que el

\section{NOTA}

Este artículo ha sido traducido del original portugués.

\section{REFERENCIAS BIBLIOGRÁFICAS}

AXT, R., 1990. A experimentaçao como estratégia para a reformulação conceitual. En prensa.

HELLMAN, W., 1989. Conceptual versus nonconceptual questions in the grading of physics students, The Physics Teacher, Vol. 26(5), pp. 383-386.

HEWITT, P.G., 1987. Conceptual physics. (Addison - Wesley: Menlo Park, CA).

LORD, F., y NOVICK, M.R., 1968. Statistical theories of mental test scores. (Addison - Wesley: Menlo Park, CA).

MOREIRA, M.A y BUCHWEITZ, B., 1987. Mapas conceiinais. (Moraes: São Paulo). aprendizaje conceptual sería una condición suficiente para convertir al alumno en un buen solucionador de problemas. El estudio que describimos sugiere claramente que el dominio de la parte conceptual del contenido es condición necesaria pero no suficiente. Probablemente, la resolución de problemas es una de las áreas más investigadas en la enseñanza de la Física después del área de conceptos alternativos. Y debe seguir siendo un área de investigación, pues, a pesar de su gran éxito, la búsqueda de conceptos alternativos todavía esta ocupada con la cuestión del cambio conceptual. Por ello, su contribución al área de resolución de problemas, sin la cual, para muchos, la Física no es Física (así como para otros, la Física sin laboratorio tampoco es Física), ha sido escasa. El presente trabajo pretende ser una contribución que lleve a corroborar, con datos numéricos, la hipótesis de que el aprendizaje de conceptos es extremadamente importante en el aprendizaje de la Física pero no es suficiente.
NOVAK, J.D. y GOWIN, D.B., 1984. Learning how to learn. (Cambridge University Press: New York).

SILVEIRA, F.L., MOREIRA, M.A. y AXT, R., 1986. Validação de um teste para detectar se o aluno possui a concepção newtoniana sobre força e movimento, Ciencia $e$ Cultura, Vol. 38(12), pp. 2047-2055.

SILVEIRA, F.L., MOREIRA, M.A. y AXT, R., 1989. Validaço de um teste para verificar se o aluno possui concepçoes cientificas sobre corrente eléctrica em circuito simples, Ciencia e Cultura, Vol. 41(1), pp. I129-1133. 